Results: Genome-wide DNA methylation analysis identified 813 DMG, including 279 hypomethylated and 534 hypermethylated. Functional classification of these genes revealed signatures associated with biological processes and pathways related to their clinical profile, including immune response, adhesion, oxidative stress and vascular signaling. Correlation and association studies showed that the methylation levels of genes related to immune response were associated with the CV-risk score, aGAPSS (CCR2, TXLNB, GLIPR), type of thrombosis (SIGLEC11, COLEC11, LRRC16A, AHSA1, TRIL) and aPL titers (CLEC4G, RGS4, HLA-DPA1, GBP6, RAET1E, HLA-G, HLA-DPA1, HLA-H, TXLNB). Besides, methylation levels of DMG related to vascular signaling and adhesion processes were associated with the presence of thrombotic recurrences (VEGFA, MAPK14, ITGA8, EPCAM, PCDHA6, DLG1) as well as with traditional CV-risk factor such as hypertension and dyslipidemia (ITGA11, DSCAM, CLEC4F, CDH4, LTBP2, PCDHB14). In addition, methylation levels of DMG genes related to oxidative stress (GP2, PGD, ADH1) were associated with microvascular endothelial dysfunction. An altered mRNA expression of some of those genes with aberrant methylation and related to increased CV-risk and thrombotic recurrences in APS was also identified. Both, abnormal methylation and transcription levels of several genes were further associated with a pathological increase of the CIMT. Finally, in vitro studies supported the role of aPLs as key players in the altered methylation and transcriptomic profiles of APS patients.

Conclusion: APS patients showed an impaired methylation profile in monocytes of genes associated with clinical features of the disease, including aPL titers, CV risk, thrombotic recurrences, endothelial dysfunction and early atherosclerosis. These results offered a map to the monocytes methylome and shed light on the pathophysiology of APS, paving the way for the development of new, more effective biomarkers and therapeutics.

Acknowledgments: Funded by ISCIII (PI18/0837 and RIER RD16/0012/0015) co-funded with FEDER.

Disclosure of Interests: Carlos Perez-Sanchez: None declared, Alejandra M. Patiño-Trives: None declared, Maria A Aguirre: None declared, Pérez Sánchez Laura: None declared, María Luque-Tévar: None declared, Iván Arias de la Rosa: None declared, Carmen Torres-Granados: None declared, Maria del Carmen Abalos-Aguilera: None declared, Pedro Seguí Azpilcueta: None declared, Javier Rodríguez: None declared, Esteban Ballester: None declared, Nuria Barbarroja Puerto Grant/research support from: ROCHE and Pfizer., Speakers bureau: ROCHE and Celgene., Eduardo Collantes Estevez Grant/research support from: ROCHE and Pfizer, Speakers bureau: ROCHE, Lilly, Bristol and Celgene, Chary Lopez-Pedrera Grant/research support from: ROCHE and Pfizer. DOI: 10.1136/annrheumdis-2020-eular.5358

\section{THU0005 VARIABILITY OF DNA METHYLATION IS A DRIVER OF LYMPHOCYTE DYSREGULATION IN EARLY RHEUMATOID ARTHRITIS.}

A. Clark ${ }^{1}$, N. Naamane ${ }^{1}$, N. Nair ${ }^{2,3}$, A. Anderson ${ }^{1}$, N. Thalayasingam ${ }^{1}$, J. Diboll ${ }^{1}$, A. Barton ${ }^{2,3}$, S. Eyre ${ }^{2,3}$, J. D. Isaacs ${ }^{1,4}$, L. Reynard ${ }^{5}$, A. Pratt ${ }^{1,4}$ ${ }^{1}$ Newcastle University Clinical and Translational Research Institute, Newcastle upon Tyne, United Kingdom; ${ }^{2}$ Versus Arthritis Centre for Genetics and Genomics, University of Manchester, Manchester, United Kingdom; ${ }^{3} \mathrm{NIHR}$ Manchester Musculoskeletal BRC, Manchester University NHS Foundation Trust, Manchester, United Kingdom; ${ }^{4}$ Musculoskeletal Services Directorate, Newcastle upon Tyne Hospitals NHS Trust, Newcastle upon Tyne, United Kingdom; ${ }^{5}$ Newcastle University Biosciences Institute, Newcastle upon Tyne, United Kingdom

Background: DNA methylation patterns differ between leukocyte subsets and mediate the impact of environmental exposures on the molecular and functional phenotype of immune cells. Besides differences in mean methylation of CpG positions amongst patients with immune mediated diseases, recent evidence indicates variability of site-specific DNA methylation also contributes to pathogenesis ${ }^{1,2}$. Objectives: To seek evidence of altered DNA methylation patterns in RA, controlling for systemic inflammation and immunotherapy use.

Methods: Patients with confirmed clinical diagnoses were enrolled from the Northeast Early Arthritis Cohort (NEAC). CD4 ${ }^{+}$and $C D 19^{+}$lymphocytes were isolated from fresh blood by positive selection prior to therapeutic immune modulation. Methylation was quantified in cell subset-specific DNA (Infinium MethylationEPIC BeadChip, Illumina) $)^{3}$. Differentially methylated positions and regions (DMPs, DMRs) between RA and non-RA patients were identified (linear modelling, filtering on $5 \%$ pairwise difference in mean DNA methylation, and DMRcate package). Next, to identify instances where methylation variance differed between comparator groups, Bartlett's test was performed using the iEVORA package, which accounts for outlier values ${ }^{4}$. Findings were controlled for technical confounders and subject to multiple test correction (FDR). A validated hypergeometric test was used to annotate enriched pathways.

Results: After sample- and probe-level quality control, CD4+ and B lymphocyte specific data were respectively available for 45 and 49 RA patients, and 64 and
81 disease controls matched for systemic inflammation (CRP, ESR). No DMPs were identified in either cell type at FDR $<0.05$ and $\Delta \beta \geq 0.05$. Only following relaxation of multiple test correction was it possible to identify DMRs in either cell type, most notably encapsulating $10 \mathrm{CpGs}$ relatively hypomethylated at the promoter of the endosome protein-encoding RUFY1 gene in CD4+ lymphocytes of RA patients $(\Delta \beta=0.076)$. By contrast, striking evidence for differential variation in DNA methylation was observed at 291 and 601 CpGs of CD4+ and B lymphocytes, respectively (exemplars depicted in Figure 1). Only 15 of these differentially variable positions (DVPs) were common to both cell types. Pathway analysis highlighted potential functional consequences of DVP associations; for example, RA-specific hypervariability implicates prostaglandinsignalling in CD4+ lymphocytes.

CD4+ T cells:
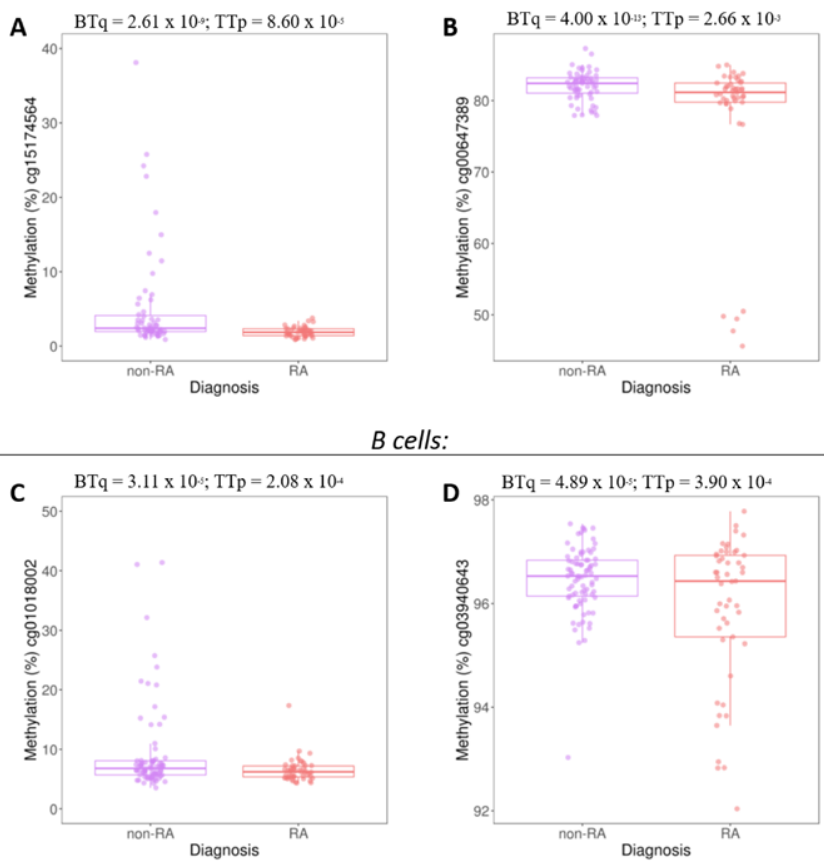

B cells:

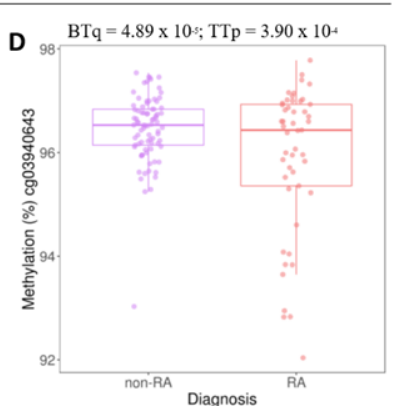

Figure 1. Plots are shown for CpGs in CD4+T cells that were found to be either (A) hypovariable or (B) hyper-variable in rheumatoid arthritis patients, with equivalent $B$ cell plots again depicting $(\boldsymbol{C})$ rheumatoid arthritis hypo-variable and $(\boldsymbol{D})$ hyper-variable positions. BTa = Bartlett's q-value, a false discovery rate adjusted measure of group differences in DNA methylation variance; TTp $=T$-test $p$-value, applied to test for differences between the group means of any positions found to exhibit differential variance between cases and controls in the Bartlett's test $(q<0.001)$

Conclusion: We highlight a role for altered variability in DNA methylation during the molecular pathogenesis of RA, and emphasise the importance of its study in relevant cell subsets.

References:

[1] Paul DS et al. Nature Communications 7, 13555 doi: 10.1038/ncomms 13555 (2016).

[2] Webster AP et al. Genome Medicine 10, 64 (2018) doi:10.1186/ s13073-018-0575-9.

[3] Clark $A D$ et al. Journal of Allergy and Clinical Immunology 2019; doi: 10.1016/j.jaci.2019.12.910

[4] Teschendorff AE et al. Nature Communications 2016; 7:12.

Disclosure of Interests: Alexander Clark: None declared, Najib Naamane: None declared, Nisha Nair: None declared, Amy Anderson: None declared, Nishanthi Thalayasingam: None declared, Julie Diboll: None declared, Anne Barton Consultant of: AbbVie, Stephen Eyre: None declared, John D Isaacs Consultant of: AbbVie, Bristol-Myers Squibb, Eli Lilly, Gilead, Janssen, Merck, Pfizer, Roche Louise Reynard: None declared, Arthur Pratt Grant/research support from: Pfizer, GlaxoSmithKlein

DOI: 10.1136/annrheumdis-2020-eular.2852

\section{THU0006 \\ ASSOCIATION BETWEEN ALTERED MICRORNA EXPRESSION AND ARTERIAL WALL REMODELING IN GIANT CELL ARTERITIS}

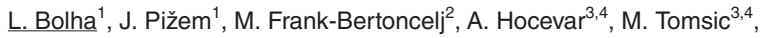
V. Jurcic ${ }^{1} .{ }^{1}$ Institute of Pathology, Faculty of Medicine, University of Ljubljana, Ljubljana, Slovenia; ${ }^{2}$ Department of Rheumatology, Center of Experimental Rheumatology, University Hospital Zurich, Zurich, Switzerland; ${ }^{3}$ Departmen 